\title{
Formación para la administración municipal basada en la participación popular protagónica
}

\author{
Porras Medina, Ada Crisey* \\ González Silva, Humberto J.**
}

\begin{abstract}
Resumen
El presente trabajo aborda la tarea de establecer referentes para la formulación de propuestas de formación para los funcionarios municipales en Venezuela, en función de la transformación de la administración de este nivel de gobierno bajo el principio de la participación popular protagónica. Para ello se realiza una revisión bibliográfica dirigida a establecer sentidos y dimensiones de la participación, a la aclaración de términos fundamentales para una propuesta formativa en el contexto venezolano, y que, asimismo, permite establecer las debilidades de la formación para la participación, generalmente centrada más en las comunidades que en los funcionarios, más en los contenidos que en las personas, desarticulada y con escasos recursos de apoyo. Las principales conclusiones apuntan a la necesidad de fundar la formación en una clara definición de participación como ejercicio de la soberanía popular, la indispensable dimensión ética y política de la formación, la afirmación de la especificidad de la administración pública, la inclusión en la formación tanto de las comunidades como de los funcionarios, el apoyo en experiencias desarrolladas en la administración y la comunidad, el requerimiento de articulación y el enriquecimiento de los enfoques educativos.
\end{abstract}

Palabras clave: Participación, Administración Municipal, Formación de funcionarios públicos.

Recibido: 15-10-07. Aceptado: 11-04-08

* $\quad$ Licenciada en Trabajo Social (Universidad del Zulia), Trabajadora Social en el Colegio Universitario Profesor José Lorenzo Pérez Rodríguez, cursante del Postgrado en Gerencia Pública de la Escuela Venezolana de Planificación. Teléfono: 0426-5164088, 0416-6205674.

E-mail: adacrisey@gmail.com

** Profesor de la Escuela de Educación de la Universidad Central de Venezuela. 
Formación para la administración municipal basada en la participación popular protagónica Porras Medina, Ada Crisey y González Silva, Humberto J.

\title{
Training for Municipal Administration Based on Popular Protagonist Participation
}

\begin{abstract}
This study takes on the task of establishing referents for formulating training proposals for municipal officials in Venezuela, in the light of the administrative transformation taking place at this level of government under the principle of popular protagonist participation. To accomplish this, a bibliographical review was performed, focused on establishing the meanings and dimensions of this participation, clarifying basic terms for a training proposal in the Venezuelan context that, at the same time, permit identifying weaknesses in training for participation, which generally centers more on communities than on the officials, more on content than on people, and which is disconnected and provided with scant support resources. Principle conclusions point to the need for basing the training on a clear definition of participation as an exercise in popular sovereignty, the indispensable ethical and political dimension of the training, an affirmation of the specificity of public administration, the inclusion of communities as well as officials in the training, support from experiences developed in administration and the community, and the requirement for interconnections and the enrichment of educational approaches.
\end{abstract}

Key words: Participation, municipal administration, training of public officials.

\section{Introducción}

La participación aparece como una preocupación mundial para dar sentido a la democracia y trazar rutas hacia un mundo justo y sustentable. En palabras del sociólogo brasileño Herbert de Souza (Betinho) ${ }^{1}$ : "La participación es uno de los cinco principios de la democracia. Sin ella, no es posible transformar en realidad, en parte de la historia humana, ninguno de los otros principios: igualdad, libertad, diversidad y solidaridad".

Así pues, la construcción de una democracia participativa y protagónica requiere una transformación del Estado a todos los niveles, pues se trata de participación en la construcción del modelo po- lítico, económico, social y cultural, de participación internacional, nacional, regional, sectorial y local. En ello se juega la suerte y la esperanza del pueblo, pues como señala Melucci (1999:11), a la estructura tradicional de las desigualdades materiales se ha sumado una nueva desigualdad asociada a "los medios con los que se define el significado de la acción, con lo que se construye la identidad individual, y colectiva", que es también y fundamentalmente uno de los significados de la participación que se opone a la exclusión, reivindicando las posibilidades de las personas y los colectivos de ser sujetos y superar la idea de una "inclusión totalmente subordinada en el consumo masivo; con la manipulación televisivo-

1 Para mayor información ver: http://www.mre.gov.br/CDBRASIL/TAMARATYMEB/espanhol/polsoc/partic/apresent/apresent. htm 
religiosa de la conciencia; con la imposición de estilos de vida que destruyen, de una vez y para siempre, las raíces de las culturas populares" (Melucci, 1999:12).

Siendo un tema vivo en distintos ámbitos, focalizamos sin embargo en este estudio la atención en el nivel local. Sobre el espacio municipal han dicho Delgado y Quintín (2006) que es el más eficaz para la participación ciudadana, y Gallicchio (2002) señala que el ámbito local es el territorio en el cual se corporizan los procesos de empoderamiento, lo cual hace imposible pensarlos desprovistos de una dimensión territorial, pues las diferentes formas, modelos, relaciones de poder, se materializan en nuestras sociedades de diferentes maneras, pero se corporizan en los ámbitos locales, en territorios concretos.

Por su parte, Coraggio (2004) apunta que lo local alude a la vida cotidiana, a un espacio de sociabilidad primaria, donde es posible para las mayorías interactuar cara a cara. De hecho, buena parte de las reflexiones y experiencias que empiezan a ser conocidas y debatidas a nivel mundial aluden a la vinculación entre participación y desarrollo local, abarcando un amplio abanico de temas.

En Venezuela, el Presidente Chávez ha asociado la participación y la creación de un efectivo poder popular a la creación y consolidación de los Consejos Comunales, en sus palabras: "los consejos comunales y el fortalecimiento del poder popular son el camino para llegar al socialismo" ${ }^{2}$.
Siguiendo este impulso se delimita el problema de este estudio al ámbito local-municipal y las preguntas apuntan a: ¿cómo construir un municipio a la medida de la participación y el poder popular?, ¿cuáles son los aspectos a atender para la construcción de este nuevo municipio? ¿Qué formación se requiere para los funcionarios municipales? ¿Cuáles deben ser las características de esta formación para que incidan efectivamente en la transformación de la administración municipal para que esté a tono con la explosión del poder comunal?

Tomando como referencia a Llona (2001), los aspectos clave para asegurar la gestión participativa del desarrollo local pueden resumirse en seis dimensiones: a) La dimensión legal, b) la dimensión organizacional, c) la dimensión formativa, d) la dimensión técnica, e) la direccionalidad, f) el clima organizacional.

Este estudio toma como foco la dimensión formativa pues ésta también tiene respuestas que ofrecer para todas las demás dimensiones y cualquier propuesta de formación debe pasearse por estas otras dimensiones. Nuestro tema se centra entonces en la construcción de referentes para propuestas formativas que contribuyan efectivamente a la transformación de la administración municipal, para contribuir efectivamente a un modelo de gestión basado en la participación protagónica de las comunidades y la construcción de poder popular.

2 Janna Corredor (02/05/2006) "Chávez destacó que consejos comunales son la vía para llegar al socialismo", en: www.abn.info.ve 
Formación para la administración municipal basada en la participación popular protagónica Porras Medina, Ada Crisey y González Silva, Humberto J.

\section{Algunas consideraciones teóricas sobre la Participación como eje de definición del nuevo Estado}

En las últimas décadas, el tema de la participación ciudadana ha alcanzado particular relevancia en las agendas de los organismos de promoción del desarrollo y ha adquirido una creciente legitimidad política y social. La participación se considera un factor clave para el desarrollo equitativo y sostenible (SD Gateway, 2006), como una nueva forma de mediación y concepción de la política (Vargas, 1998) y también como una alternativa creativa frente a las tradicionales formas de implementación de políticas públicas (Vargas, 1998).

La Constitución de la República Bolivariana de Venezuela (CRBV, 1999) hace de la participación protagónica uno de los ejes de la refundación de la República y entiende la participación como un derecho y un deber de los ciudadanos. Esta idea está presente en la Exposición de Motivos de la CRBV y se reafirma en los artículos 62 (sobre el derecho a la participación), 70 (sobre los medios de participación), el 132 (sobre el deber de participar) y el 184 (sobre la descentralización de la participación).

La participación, según Coraggio (2004:33), hace referencia a "tomar parte", es decir, formar parte de acciones, procesos, decisiones colectivas dentro de espacios conformados por ciudadanos organizados para incidir sobre una realidad que se quiere cambiar. El Presidente de la República Bolivariana de Venezuela, Hugo Rafael Chávez Frías, la ubica en el contexto de la conformación del nuevo modelo de sociedad planteado por el Gobierno Bolivariano, así lo señala en su discurso en las Naciones Unidas en Septiembre de 2004, "Si queremos acabar con la pobreza demos poder a los pobres". Para ese momento el presidente señaló:

Si los pobres no participan en las decisiones que modelan la vida social, si no son elementos activos en la lucha contra la pobreza, si no existe un poder político que con firmeza haga de los pobres y del combate a la pobreza el centro de sus acciones, si no son los pobres parte importante del poder político ellos mismos, los recursos financieros podrán ser paliativos, pero nunca serán soluciones (Chávez, 2004b:12).

Esta voluntad de impulsar la participación como eje de definición del nuevo estado se reafirma en la formulación de "El nuevo mapa estratégico" (2004a) que define como objetivo estratégico: "Avanzar aceleradamente en la construcción del nuevo modelo democrático de participación popular" y, más aún en la definición del Quinto Motor Constituyente: La Explosión del Poder Comunal. Sin embargo, como ha señalado también el presidente, para avanzar hacia una democracia participativa y protagónica, se encuentran serios obstáculos en el propio Estado.

El Estado no parece ir al ritmo de transformación que exige la construcción de una democracia participativa. Como señala Kliksberg (1992:35), refiriéndose a la gestión social en particular:

... la burocracia social tradicional tiene fuerte resistencia a dar lugar real a las poblaciones asistidas. Construye una telaraña de racionalizaciones y mitos respecto de las consecuencias caóticas 
que ello tendría para los programas, obstruye la participación y finalmente, en algunos casos, adjudica a los asistidos un bajo interés en participar, cuando ha hecho todo lo posible por desalentar su intervención (...) No se haya capacitada para la participación, ni maneja el instrumental técnico necesario, por lo que la misma constituye una sobrecarga para sus tareas.

Fenómeno reafirmado en nuestra coyuntura por el presidente Chávez cuando cuenta por ejemplo que: "Me ha llegado información de que algunos alcaldes nuestros lo que hacen más bien es frenar y entran en conflicto con las comunidades porque no quieren que la gente participe..." (Chávez, 2004b:41).

Por su parte, El Troudi, Harnecker y Bonilla (2005:23) apuntan que:

Los funcionarios de gobierno (local, regional o nacional), incluidos los revolucionarios, suelen aproximarse a las comunidades con una precaria preparación para ello (...) las organizaciones de base y los líderes comunitarios son muy sensibles cuando descubren la improvisación con la cual actúa la burocracia ante los ciudadanos...

Estas tres versiones sobre los obstáculos para la participación que surgen desde el Estado permiten caracterizar como elementos centrales de la situación: la lógica heredada de la burocracia, el temor a la participación por la consiguiente pérdida de poder para los funcionarios y aparatos del Estado y la debilidad en la capacitación de los funcionarios, así como la rigidez del dogmatismo, definido por El Troudi, Harnecker y Bonilla (2005:30) como:
... razonar con recetas prefabricadas frente a los procesos sociales y políticos sin analizar la situación real del país que se quiere transformar (...) el dogmatismo no reconoce la riqueza de caminos y propuestas que nutren la transformación social que emprende la gente, siempre busca moldes para encauzar la realidad a ellos.

A estos elementos podría sumarse la inexistencia de modelos claros de gestión basados en la participación social, que dificultan establecer referentes para la acción del Estado que puedan ser evaluados.

\section{Formulación de Propuestas de Formación}

Tomamos tres focos para la discusión de los referentes para la formulación de propuestas de formación dirigidas a la transformación de la administración municipal con base a la participación popular. En primer lugar, la definición o más bien las definiciones sobre participación, desarrollando las particulares implicaciones de la participación como base del poder popular. En segundo lugar, se aborda la especificidad de la administración pública municipal, resaltando las necesarias distinciones con la gerencia privada, en aras de prevenir la importación acrítica de conceptos, que aún en tiempos de revolución socialista suele practicarse cuando se habla de gestión y formación para la gestión. Por último se discuten los enfoques, métodos y contenidos de la formación, partiendo de la revisión de experiencias y propuestas latinoamericanas. 
Formación para la administración municipal basada en la participación popular protagónica Porras Medina, Ada Crisey y González Silva, Humberto J.

\subsection{Participación, gestión municipal y poder popular}

Desde una óptica de gestión se han planteado distintas razones que hacen de la participación una herramienta fundamental para el mejoramiento de la gestión pública. Un resumen de estas ventajas nos lleva a una lista como la siguiente (apoyada en buena parte en los planteamientos de Millaleo y Valdes (2002:3):

- La participación puede incrementar la eficacia y la eficiencia de las actividades de desarrollo mediante el involucramiento de los recursos y destrezas de quienes participan permitiendo además hacer un mejor manejo de las externalidades asociadas.

- Permite el incremento en la efectividad de las actividades, reconociendo que ellas están basadas en el conocimiento y comprensión de los problemas sociales.

- La participación ayuda a construir e instalar capacidades y desarrollar habilidades de aquellos quienes se encuentran inmersos dentro del proceso.

- La participación puede incrementar la cobertura en la medida en que los involucrados asuman ciertas responsabilidades, a su vez, que tomen otras responsabilidades no contempladas inicialmente.

- La participación puede contribuir a mejorar la planificación y así ampliar los beneficios.

- La participación logra la identificación de los ciudadanos involucrados con los proyectos, así como también el compromiso al momento de su ejecución y control.
- La participación es importantísima para asegurar la sostenibilidad de los proyectos.

- La participación puede ampliar y mejorar las relaciones entre los ciudadanos y entre estos y los funcionarios públicos.

- La participación aumenta la autoestima popular.

- La participación también puede lograr la superación de las divergencias y limitar las divisiones entre grupos o sectores.

Desde el punto de vista estratégico, la participación popular es clave de la construcción de la nueva sociedad que plantea la Constitución de la República Bolivariana de Venezuela, la creación de ese otro mundo posible, exigido reiteradamente desde el Foro Social Mundial. Como señala Millaleo y Valdes (2002) independientemente de las ventajas de la participación para los procesos de desarrollo esta debe constituir un objetivo estratégico de dichos procesos:

... es posible afirmar que esta consideración es de carácter normativo, en cuanto, cualquiera sea la situación de los potenciales involucrados y su relación con los niveles decisionales, es posible sostener que es, al menos de la perspectiva de la ética política, obligatorio considerar la opinión de los involucrados (...) la idea de la democracia participativa que está detrás de las demandas de participación ciudadana y de empoderamiento deriva sin lugar a dudas del viejo ideal de que los sujetos no solo pueden ser destinatarios sino que deben ser autores de las decisiones que afecten sus condiciones de 
vida, pues de otra manera perderían su autonomía, condición que los define como ciudadanos. Millaleo y Valdes (2002:4).

De esta manera el carácter normativo de la participación crea para las instituciones públicas la obligación de impulsar el empoderamiento de las comunidades:

El empoderamiento en el contexto de una estrategia social es un proceso selectivo, consciente e intencionado que tiene como objetivo la igualación de oportunidades de los actores sociales. El criterio central es de transformación de sectores sociales excluidos en actores, y de nivelación hacia arriba de actores débiles. El empowerment ha sido definido como el proceso por el cual la autoridad y la habilidad se ganan, se desarrollan, se toman o se facilitan (Millaleo y Valdes, 2002:4).

El aporte que podamos hacer entonces para la transformación de las administraciones municipales y el fortalecimiento de sus capacidades para promover la participación, puede justificarse entonces tanto desde el punto de vista de la funcionalidad de la gestión como desde el punto de vista político que hemos señalado. Mas el enfoque, funcional o político, que se adopte tiene claras consecuencias para los alcances y significados de la participación y en consecuencia para las propuestas de formación.

Coraggio (2004) diferencia tres niveles de participación popular: el primero concentrado en la institución familiar, en la residencia, en el lugar de trabajo y la participación en el mercado, vinculado a las condiciones de reproducción más elementales de la vida y a la esfera de la cotidianidad. Un segundo nivel tiene que ver con las organizaciones populares, como los sindicatos, movimientos reivindicativos, cooperativas de producción o de vivienda, organizaciones barriales, etc. $Y$ un tercer nivel que es el de la sociedad en cualquiera de sus ámbitos (local, regional, nacional, internacional):

... En este nivel se da la producción y eventualmente la transformación de la sociedad y del Estado, una relación entre sociedad que muy esquemáticamente veremos como necesaria. Mientras la sociedad sea dividida, heterogénea, conflictiva, la forma Estado será necesaria para generar una comunidad ilusoria en medio de esa heterogeneidad, de ese conflicto. Este es el mundo de la política (Coraggio, 2004:36).

El autor describe como cada uno de estos niveles es un escenario de lucha social y conflicto de intereses y de visiones. Desde los sectores poderosos que propugnan la reducción del primer nivel a las relaciones del egoísmo características del mercado, intentan instrumentalizar la participación en el segundo nivel y eliminar ideológicamente el tercer nivel, concibiendo a los sectores populares que actúan en él como meros grupos de interés parciales, de manera que se les dificulte estructurarse en una propuesta global de cambio. Desde los sectores populares que intentan reivindicar el primer nivel como espacio de convivencia y realización de nuevos valores, al segundo como espacio de articulación para la incidencia en los asuntos públicos y al tercer nivel como aquel en que ha de propugnarse la transformación del modelo de opresión, exclusión y explotación.

Coraggio (2004) resalta que hay una batalla por los sentidos de la partici- 
Formación para la administración municipal basada en la participación popular protagónica Porras Medina, Ada Crisey y González Silva, Humberto J.

pación popular e identifica tres sentidos en pugna: participar en función de la reproducción, participar como legitimar o deslegitimar el poder constituido y el de la participación como un ejercicio autónomo de la soberanía popular.

Utilizando estas categorías y tomando como base las observaciones de Coraggio, puede establecerse la siguiente vinculación entre los niveles y sentidos de la participación: En el primer sentido (la participación en función de la reproducción), la participación en el primer nivel se entiende como acción individual en el mercado, en el segundo nivel como organización para resolver problemas que no pueden resolverse por vías de mercado y en el tercero, como organización para hacer valer intereses parciales o sectoriales.

En el segundo sentido (participar como legitimar o deslegitimar el poder constituido), la participación se asocia a un sentido pasivo: el voto o el no voto, la protesta o el apoyo. En el tercer sentido, la participación se entiende como:

... ejercicio autónomo de la soberanía popular, por un proyecto de nueva sociedad como marco para revolucionar la vida cotidiana, para jerarquizar los derechos humanos. Esto sería un tercer nivel, pero activo (Coraggio, 2004:36).

Una representación de la relación entre niveles y sentidos de la participación popular se ilustra en el Cuadro 1, el cual nos puede servir para identificar dife- rencias en los discursos sobre la participación y el poder popular.

En el proceso de cambios que vive Venezuela, se plantea claramente el debate entre los sentidos y niveles de la participación. Por una parte, el Quinto Motor: La explosión del Poder Comunal fue anunciado por el Presidente Chávez como una dimensión fundamental de la nueva etapa de la Revolución Bolivariana, directamente alineada con la construcción de una sociedad socialista. Este Quinto Motor se identifica con el ejercicio autónomo de la soberanía popular para la construcción de una nueva sociedad y ha sido definido por Jorge Rodríguez, Vicepresidente Ejecutivo de la República Bolivariana de Venezuela, en los siguientes términos:

Los Consejos Comunales, el Poder Comunal es el nuevo Gobierno, es la nueva forma de Gobierno que nos queremos dar y ha sido enfático y ha sido claro el comandante Chávez cuando ha dicho hay que repartir, hay que dar el Poder al pueblo y es por eso que resulta tan importante esta explosión del Quinto Motor ${ }^{3}$.

El camino para la constitución del Poder Comunal en el nuevo Gobierno es descrito como una trayectoria explícita:

El futuro será para que los ministros y los viceministros el Vicepresidente, y el Gobierno todo y los alcaldes y los gobernadores, vayamos a preguntarles a ustedes, dónde quieren las obras, vaya-

3 Jorge Rodríguez (2007) Explosión del Quinto Motor, discurso pronunciado en Acto con motivo de la explosión del Quinto Motor Constituyente, en el Parque Los Caobos, Caracas, el 28 de febrero de 2007. Disponible en: http://www.vicepresidencia.gov.ve/alocucion.asp?numn=11 


\begin{tabular}{|c|c|c|c|}
\hline \multicolumn{4}{|c|}{$\begin{array}{c}\text { Cuadro } 1 \\
\text { Niveles y sentidos de la participación }\end{array}$} \\
\hline & $\begin{array}{l}\text { Primer nivel: } \\
\text { Nivel doméstico }\end{array}$ & $\begin{array}{c}\text { Segundo nivel: } \\
\text { Organización } \\
\text { reivindicativa }\end{array}$ & $\begin{array}{l}\text { Tercer nivel: } \\
\text { Nivel social }\end{array}$ \\
\hline $\begin{array}{l}\text { Primer sentido: } \\
\text { Participar en función } \\
\text { de la reproducción }\end{array}$ & $\begin{array}{l}\text { Participación individual } \\
\text { en el mercado }\end{array}$ & $\begin{array}{l}\text { Resolución de } \\
\text { problemas inmediatos } \\
\text { que no pueden } \\
\text { resolverse por medio } \\
\text { del mercado }\end{array}$ & $\begin{array}{l}\text { Expresión de intereses } \\
\text { sectoriales }\end{array}$ \\
\hline $\begin{array}{l}\text { Segundo sentido: } \\
\text { Legitimar o } \\
\text { deslegitimar }\end{array}$ & & $\begin{array}{c}\text { Presión o colaboración } \\
\text { con el poder por medio } \\
\text { de movilizaciones, } \\
\text { negociaciones }\end{array}$ & $\begin{array}{l}\text { Participación o no } \\
\text { como votante. } \\
\text { Movilización política } \\
\text { a favor o en contra }\end{array}$ \\
\hline $\begin{array}{c}\text { Tercer sentido: } \\
\text { Ejercicio autónomo } \\
\text { de la soberanía } \\
\text { popular }\end{array}$ & $\begin{array}{l}\text { Marco para } \\
\text { revolucionar la vida } \\
\text { cotidiana }\end{array}$ & $\begin{array}{l}\text { Organización de base } \\
\text { para el ejercicio del } \\
\text { poder en la esfera } \\
\text { local o sectorial }\end{array}$ & $\begin{array}{l}\text { Construcción de una } \\
\text { nueva sociedad }\end{array}$ \\
\hline
\end{tabular}

Fuente: Elaboración propia.

mos a preguntarles a ustedes cómo se organiza el presupuesto, vayamos a preguntarles a ustedes y sobre todo vayamos a exigirles a ustedes que controlen el desarrollo de los presupuestos, que controlen la acción del Gobierno, esa es la función del Poder Comunal, no es nada más, amigas y amigos compatriotas, no es nada más unas organizaciones para resolver los problemas específicos de su comunidad, también es para eso pero no podemos quedarnos allí, no podemos quedarnos solamente en Consejos Comunales aislados los unos de los otros, tenemos, estamos obligados a que esta red que explota hoy, que se difumina por todo el país, a partir del próximo fin de semana, esa red se mantenga, se sostenga, se consolide, para que un futuro muy cercano, no hablemos de Consejos Comunales solamente, sino de Federaciones de
Consejos Comunales, de territorios comunales, de comunidades y en un futuro, ojalá cercano, del Estado Comunal (Rodríguez, 2007).

En contraste, otras visiones sobre la participación, interpretan la Explosión del Poder Comunal, como un proceso de diagnóstico y de elaboración de propuestas, que permitan mejorar el funcionamiento de los consejos comunales; pensando en un proceso mediado y guiado por los funcionarios del gobierno en aras de cumplir con un mandato legal y del cumplimiento de requisitos.

Así pues, una formación dirigida a tener un Estado capaz de afrontar el reto de desarrollar el Poder Popular (tal como ha quedado explícito en las nuevas denominaciones de los ministerios, por ejemplo) tiene que afrontar en primer término el tema de las conceptualizaciones y participar de la lucha que describe Coraggio 
Formación para la administración municipal basada en la participación popular protagónica Porras Medina, Ada Crisey y González Silva, Humberto J.

o de la que, en palabras de Fidel Castro, es una batalla de las ideas.

Otras dificultades deben encararse en el impulso a la participación, la lista que presentan El Troudi, Harnecker y Bonilla (2005) incluye: 1) el escepticismo y la apatía, 2) la cultura clientelar y de otorgamiento de favores, 3) el burocratismo, 4) el verticalismo y autoritarismo, 5) escasez de tiempo, 6) incapacidad de escuchar, 7) intolerancia, 8) desconfianza en el pueblo, 9) improvisación de los funcionarios del gobierno, 10) dirigentes sabelotodo, 11) el espontaneismo de las comunidades, 12) el asambleismo, 13) formalismo declarativo, 14) perfeccionismo, 15) sectarismo, 16) dogmatismo, 17) uso de calificativos peyorativos y 18) exceso de pragmatismo.

Estos autores plantean conjuntamente valores y nuevas sensibilidades que es necesario desarrollar: Elevación de la autoestima popular, respeto a las diferencias, superación de las divisiones y politización, entendiendo por esta última que: "La participación popular protagónica en las distintas instancias de gobierno hace que las personas involucradas en ella crezcan en conciencia política" (EI Troudi, Harnecker y Bonilla, 2005:34).

En este sentido se pone de relieve que:

... el ejercicio de la política ha sido sustraído del ejercicio cotidiano de la gente siendo arrebatado por un sector que se llama a sí mismo profesional de la política y que, en definitiva, es el úncio que se siente con derecho a opinar, a decidir (El Troudi, Harnecker y Bonilla, 2005: 35).

Igualmente que "los cuadros políticos" -lo cual es asimilable a los funciona- rios públicos sin mayor pérdida de sentido- no pueden ser cuadros con mentalidad militar (pues hoy no se trata de conducir un ejército, aunque a veces esto sea necesario) ni tampoco demagogos populistas (porque no se trata de conducir un rebaño), sino que deben ser "pedagogos populares", capaces de potenciar toda la sabiduría que existe en el pueblo.

Los autores citados ponen de relieve también la necesidad de desarrollar "herramientas para la participación", entre las cuales mencionan tres de especial importancia en el ámbito municipal: el diagnóstico participativo, el presupuesto participativo y la contraloría social.

\subsubsection{Participación en el ámbito} municipal

La participación ciudadana aparece como un elemento y un propósito que debe definir la gestión municipal. El ámbito local, además, es considerado por diversos autores como el más propicio para el ejercicio de la participación, pues, como señala Fisher (1993), el poder local se realiza en un espacio delimitado donde ocurre la formación de identidades y prácticas sociales específicas.

Distintos autores latinoamericanos reportan experiencias de participación en el ámbito local. Cabrero Mendoza (1995), por ejemplo, señala que en México se observan experiencias de gobiernos municipales -de diversos partidos en el poderque fomentan nuevos sistemas y estructuras administrativas, que fortalecen la institucionalidad de este nivel de gobierno y que promueven la participación ciudadana como parte de las formas cotidianas de gestión de programas y políticas. En Perú, la Ley Orgánica de Municipali- 
dades establece, los aspectos que dan sustento a la participación en la gestión municipal. Así, parte de la definición del gobierno local radica en su carácter de entidad que canaliza la participación ciudadana en los asuntos públicos, con el objetivo de cumplir parte de su Misión (ver http://www.municipioaldia.com.pe/ participacion.php).

Entre las experiencias más conocidas de participación popular en la gestión local deben considerarse las de Villa El Salvador, en Perú y la de la municipalidad de Porto Alegre, Brasil.

Cortázar y Lecaros (2003) reseñan ampliamente la experiencia del presupuesto participativo en Villa El Salvador, apuntando como

... la ejecución de las obras y la rendición de cuentas fueron posibles gracias a un conjunto de cambios y adaptaciones que los funcionarios municipales introdujeron en su labor. Pese a que dichos cambios fueron improvisados, generaron nuevas capacidades organizativas en la administración municipal. La Dirección de Desarrollo Urbano fue tal vez aquella que mayores transformaciones vivió. Su Director reconoce cambios importantes en la interrelación de los miembros de la Dirección: "Creo que se replanteó el tema del equipo. (Este llegó ser) un equipo más horizontal, un equipo en el que no había temas (que eran de) unos y otros temas (que eran) de otros, (hubo) una (mayor) cooperación interna.." Sin embargo, y a juicio del mismo funcionario, los cambios más

4 http://habitat.aq.upm.es/bpn/bp049.html importantes se produjeron en el puesto de trabajo de los ingenieros, que se amplió mediante nuevas responsabilidades, capacidades y posibilidades de desarrollo:

Observación vinculada al planteamiento de Coraggio (2004) en el sentido de que el encuentro entre el saber técnico y el saber de la gente es un proceso de aprendizaje mutuo, cuya realización es fundamental para la construcción de una experiencia de gobierno local efectivamente participativo.

Por su parte, una reseña de la experiencia del presupuesto participativo en la ciudad de Porto Alegre, Brasil, publicada en la Biblioteca Ciudades para un Futuro más Sostenible ${ }^{4}$, la resume apuntando que

La historia de la elaboración y de la ejecución de los presupuestos públicos en Brasil está marcada por graves deformaciones relacionadas con la concentración de poder, el despilfarro de los recursos, los asuntos políticos y la corrupción. En Porto Alegre esta historia ha cambiado. Hace siete años, el Ayuntamiento de Porto Alegre creó un sistema innovador y revolucionario para formular y hacer el seguimiento del presupuesto municipal.

En este sistema, llamado Presupuesto Participativo, no participan únicamente los técnicos y las autoridades del gobierno municipal, decidiendo sobre la recaudación de impuestos y el gasto de los fondos públicos, encerrados en sus oficinas. Es la población, a través de un 
Formación para la administración municipal basada en la participación popular protagónica Porras Medina, Ada Crisey y González Silva, Humberto J.

proceso de debates y consultas, quien determina y decide la cuantía de los ingresos y los gastos, así como dónde y cuándo realizar las inversiones, cuáles son las prioridades y cuáles son los planes y acciones que debe llevar a cabo el Gobierno.

Conforme a la misma fuente, el Presupuesto Participativo ha demostrado que la administración transparente y democrática de los recursos es la única manera de evitar la corrupción y el despilfaro de los fondos públicos. A pesar de opiniones contrarias de los tecnócratas, la participación popular ha demostrado que el gasto es eficaz y efectivo donde tiene que serlo, y que los logros conseguidos en las obras públicas y en otros ámbitos son importantes para la población. Desde su inicio, los proyectos decididos mediante el Presupuesto Participativo, suponen inversiones superiores a los 700 millones de dólares, principalmente en infraestructura urbana y en la mejora del nivel de vida de la población.

\section{Administración y gestión pública, el contraste necesario con la gerencia privada}

\subsection{Administración pública y gestión privada}

La administración pública, en su concepto más amplio es todo el sistema de gobierno, todo el conjunto de ideas, actitudes, normas, procesos, instituciones, y otras formas de conducta humana que determinan cómo se distribuye y se ejerce la autoridad política y cómo se atienden los intereses públicos.

Los fines de la administración pública son establecidos en el artículo 141 de la Constitución de la República Bolivariana de Venezuela:

"La Administración Pública está al servicio de los ciudadanos y ciudadanas y se fundamenta en los principios de honestidad, participación, celeridad, eficacia, eficiencia, transparencia, rendición de cuentas y responsabilidad en el ejercicio de la función pública con sometimiento pleno a la ley y al derecho".

Tenemos que entender entonces, que la administración pública exige el desarrollo de una conceptualización propia y distinta de la gerencia privada.

La expresión "gerencia privada" rara vez puede encontrarse en la literatura, ello no es casual pues sólo se habla de gerencia privada en oposición a la gerencia pública. La gerencia es en su origen privada, se refiere a una modalidad de gestión inédita aparecida con el desarrollo de la sociedad industrial (Guerrero, 2005), en la cual surgen nuevos tipos de administradores, los jerarcas industriales que asumen el nombre de gerentes.

Mientras que la gerencia pública se basa en la gerencia privada. De hecho, el prestigio logrado por la actividad gerencial y la mitificación de la gerencia ha hecho en muchos casos que se importen acríticamente las ideas de la gerencia privada a la gestión pública. Señala Guerrero (2005) que:

Durante el siglo $X X$, principalmente a partir de los años veinte, existió la pretensión dentro de algunos círculos académicos de la administración privada, así como en algunos ámbitos de la administración pública, por asimilar en una única teoría ambos campos del saber. En años recientes, merced a la irrupción del neoliberalismo, esa ten- 
dencia tendió a tomar nuevos bríos.

Empero, esa pretensión experimentada en los Estados Unidos, escasamente ha tenido éxito en otros países, principalmente debido a la persistencia dominante de la tradición política y jurídica románica en el estudio de los asuntos políticos y administrativos.

Las diferencias entre el ámbito de la administración pública y la empresa privada han sido señaladas desde distintos puntos de vista. Stiglitz (1989) señala dos características definitorias del ámbito estatal: La pertenencia universal (todos los ciudadanos están bajo la jurisdicción del Estado) y el poder de coacción (el Estado tiene el poder de introducir impuestos, legislar, prohibir y castigar). Ninguna de estas funciones es asimilable a la relación entre una empresa y un cliente.

Estas diferencias esenciales se expresan en un conjunto de características que hacen de la gestión pública una actividad de naturaleza diferente a la gerencia privada:

a) "Las instituciones públicas no están, como las privadas, buscando conquistar a la clientela, ni retenerla. La relación es más bien la contraria: la administración pública se debe a la ciudadanía, vive de sus aportes como contribuyentes, independientemente del uso que cada ciudadano les dé a los servicios públicos" (Tohá y Solari, 1996).

b) "El ciudadano goza de derechos que lo hacen superar cualquier condición de consumidor o cliente, pues para tales derechos existen obligaciones ante el Estado" (Guerrero, 2005).

c) "Los gestores públicos deben actuar de conformidad con el interés públi- co" (CLAD, 2007), el sentido de la gestión estatal no es la ganancia o el lucro, por lo cual no puede juzgarse la gestión exclusivamente a partir de los balances ni otros criterios contables.

d) Los objetivos de la gestión pública son complejos y dinámicos pues requieren de apoyos políticos y consensos (Sulbrandt, 1992), por ello "la administración pública se caracteriza esencialmente por ser difusa, en tanto que la administración privada por ser concentrada" (Guerrero, 2005).

e) En el proceso decisorio en el sector privado, el número de participantes es restringido, y la capacidad para tomar decisiones más rápidas es mayor. El gobierno, por su parte, toma sus decisiones de acuerdo con procedimientos democráticos, que involucran un número mayor de actores y una mayor demora en el proceso decisorio, sobre todo porque tal sistema presupone el control mutuo entre los poderes y la fiscalización de la oposición (Guerrero, 2005).

Estos elementos permiten distinguir la gestión pública y la gerencia privada como campos diferenciados. Sin embargo como se ha dicho, la desvalorización de la función pública y la fiebre privatizadora característica de los finales del siglo XX, ubicaron la gerencia pública bajo la égida de los criterios de la gerencia privada y replantean la necesidad de transformar la administración del estado para asimilarla a los supuestos del mercado.

Esta tendencia se entrecruza con las exigencias de cambio del modelo burocrático tradicional, hacia mecanismos $y$ criterios de gestión que hagan frente a la complejidad y la incertidumbre, a las nece- 
Formación para la administración municipal basada en la participación popular protagónica Porras Medina, Ada Crisey y González Silva, Humberto J.

sidades de democratización y participación, a los requerimientos de una gestión orientada hacia la justicia social y la construcción de un desarrollo humano integral sustentable. En estos dos caminos se debate la definición del gerente público.

\subsection{La Administración Municipal}

Conforme a la Constitución de la República Bolivariana de Venezuela, los municipios constituyen la unidad política primaria de la organización nacional y gozan de personalidad jurídica y autonomía dentro de los límites de la Constitución y de la ley (artículo168), este mismo artículo establece que:

Las actuaciones del Municipio en el ámbito de sus competencias se cumplirán incorporando la participación ciudadana al proceso de definición y ejecución de la gestión pública y en el control y evaluación de sus resultados, en forma efectiva, suficiente y oportuna, conforme a la ley.

La autonomía de los municipios se especifica en la Ley Orgánica del Poder Público Municipal en su artículo 3:

... es la facultad que tiene el Municipio para elegir sus autoridades, gestionar las materias de su competencia, crear, recaudar e invertir sus ingresos, dictar el ordenamiento jurídico municipal, así como organizarse con la finalidad de impulsar el desarrollo social, cultural y económico sustentable de las comunidades locales, y los fines del Estado.

$Y$ dentro de las funciones a cumplir en el territorio municipal se encuentran en el Artículo 4, Parágrafo 9 "Impulsar y promover la participación ciudadana, en el ejercicio de sus actuaciones".

\section{La formación de funcionarios públicos para la participación}

La revisión de experiencias de formación vinculadas a la participación en el ámbito local de varios países latinoamericanos, nos permiten también identificar algunas lecciones. Se presentan algunas experiencias latinoamericanas en formación para funcionarios públicos y participación.

Iglesias y Tabares (2004), en su trabajo La Universidad y la Formación de Recursos Humanos como Soporte del Perfeccionamiento de la Gestión Local, afirman que la formación en Administración Pública se ha convertido en un campo incuestionable de desarrollo profesional en el que desempeñan un activo papel las Instituciones de Educación Superior y a partir de este supuesto analizan la experiencia acumulada en la Universidad de la Habana durante los 10 años anteriores para dar respuesta a necesidades en este campo. En este trabajo se identificaron un grupo de problemas vinculados con la formación, como por ejemplo: rezago y obsolescencia de los planes y programas de estudio con relación a los problemas actuales; debilidad de los programas gubernamentales de capacitación; disociación entre enseñanza y capacitación; ausencia de educación continua; uso excepcional de la enseñanza a distancia; carencia de una formación profesional progresiva; desarticulación docencia-investigación; predominio de la cátedra magistral e insuficiente; insuficiencia de profesores de carrera y escasez de insumos bibliográficos nacionales. 
Destaca en este trabajo, la realización de una Determinación de Necesidades de Aprendizaje en los Consejos de Administración Municipal de La Habana; el diseño y realización de programas de formación de entrenadores y el desarrollo de consultorías o asesorías en entidades de la administración local.

Entre las conclusiones más resaltantes de este trabajo se encuentran: a) la necesidad de incorporar a la formación temas de actualidad e interés (Gestión del Medio Ambiente, Mujeres Directivas, Dirección Estratégica. Administración por Objetivos. Gestión de Recursos Humanos, Sistema de Cuentas Nacionales, Protección del Consumidor, Ética y Responsabilidad Pública, Cultura Cubana, problemas de Política Internacional), b) la importancia de la combinación de estrategias de formación (acciones de capacitación, trabajo de consultoría, cursos a distancia, asesorías especializadas, desarrollo de encuentros de intercambio de experiencias) conforme a las necesidades de formación identificadas y c) la necesidad de la ampliación y sistematización de la actividad de investigación científica en esta esfera, mediante la organización o participación en eventos locales, nacionales o internacionales, así como a través de las relaciones de colaboración e intercambio internacional.

Aristizábal y otros (2001), en su trabajo de grado: Sistematización de Experiencias de Veedurías Ciudadanas y Control Social en la Administración Pública Estrategias de Participación Ciudadana, plantean que el estado colombiano en la Constitución Nacional de Colombia se estableció como principio fundamental la participación, pero esta práctica social se reconoce por sus resultados y en informes, pero su práctica y experiencia cotidiana no se ha recopilado y por lo tanto no se puede precisar el ejercicio metodológico interno con que se han abordado esas realidades, sus alcances y obstáculos, por lo que el Departamento Administrativo de la Función Pública manifestó su interés a la Universidad de la Salle, de rescatar por medio de la sistematización elementos metodológicos que permitan desarrollar novedosas estrategias de formación, espacios y procedimientos administrativos para pasar de la norma a la práctica y así promover la gestión pública en torno a la demanda de la sociedad civil, es decir, aprender de la práctica, tanto de lo exitoso como de aquellos aspectos que limitan el actuar social.

Entre las conclusiones más resaltantes de este trabajo se encuentran que la capacitación promovida por el estado colombiano se ha concentrado principalmente en aspectos legales y técnicos de la veeduría ciudadana, haciendo menos énfasis en la formación pedagógica y ética, además se dirige a grupos ciudadanos y en mínima proporción a funcionarios de entidades públicas.

Díaz, Rivera y Krraushaar (2002) en su artículo Nuevas Estrategias de Formación Profesional para la Modernización del Estado en Chile, plantean la necesidad de la modernización del estado, por lo que algunas Universidades Públicas de Chile desarrollan programas de formación profesional dirigidos a los trabajadores del sector público para incentivar el desarrollo de competencias y destrezas de estos profesionales. El desarrollo de programas de formación profesional para funcionarios públicos es aborda- 
Formación para la administración municipal basada en la participación popular protagónica Porras Medina, Ada Crisey y González Silva, Humberto J.

do analizando las principales tendencias y cambios que se han experimentado en los sistemas de formación profesional y técnicas desarrolladas por la Universidad de los Lagos. Otra variable que desarrollan es la relación que existe entre las competencias profesionales de los funcionarios públicos, los intereses de éstos y los discursos del estado en su proceso de modernización.

Entre las conclusiones de este trabajo se encuentran: a) la valoración del aumento del capital humano como estrategia viable para acercarse al desarrollo ha ocupando un lugar importante en los sistemas de formación profesional; b) la formación de los trabajadores debe ser continua a lo largo de toda su vida; c) es crucial la alta valoración de las competencias desarrolladas y un alto sentido de pertenencia con sus trabajos; d) es necesario profundizar las líneas de investigación respecto a los procesos pedagógicos a los efectos de lograr la profesionalización y afinar las competencias a desarrollar.

Fontdevila (2002), plantea críticas provenientes de diversos sectores que ponen en tela de juicio la credibilidad y la pertinencia de las gestiones gubernamentales, así como las potencialidades del sistema democrático. Muchos de estos planteamientos encuentran su sustento en la carencia de dirigentes y funcionarios preparados y formados específicamente para desarrollar sus funciones, por lo que los Programas de Formación para el Sector Público, las Escuelas de Administración y las Universidades en General, han tomado nota de su enorme responsabilidad en la contribución a la superación de esta crisis y en la consi- guiente oportunidad que se abre a la educación argentina del Nuevo Milenio.

Así mismo, se hacen necesarias la creatividad y la innovación de los funcionarios, acompañadas con nuevas competencias en el orden de atender la complejidad y el cambio permanente de los factores intervinientes dentro del sector público y de una carrera de formación para dirigentes sociales y políticos, que debe tener muy en cuenta estos nuevos requerimientos: No bastan las herramientas tradicionales de Administración Pública, es necesario un nuevo equipamiento de gestión y planificación para el cambio y la incertidumbre.

En función de lo anterior, plantea un sistema de Educación a Distancia que permita democratizar y hacer realidad el lema Calidad + Formación: un cambio en la gestión política de Argentina. En ese país, como en el resto del mundo, la educación a distancia clásica, basada en el aprendizaje autónomo de los alumnos mediante libros de texto, con un mínimo contacto con el docente, está dando paso a nuevas formas de formación cuya característica principal estriba en concentrarse prioritariamente en el aprendizaje y en el alumno y utilizar ampliamente las Nuevas Tecnologías de la Información y Comunicación.

Álvarez et al. (2005) presentan en el informe final del Proyecto de actualización curricular en materia de integración para los cursos de licenciaturas, postgrados y capacitación de funcionarios públicos en la comunidad andina, algunos asuntos clave para que pese a no referirse al tema de la participación son de interés para este estudio, entre ellos: a) la propuesta de una metodología para la 
continua actualización curricular en materia de integración, y b) una perspectiva metodológica para la etapa de identificación de necesidades, el proceso mismo de diseño curricular y su evaluación.

En este informe se presentan como elementos fundamentales para la actualización curricular: a) concederle más importancia al cursante como sujeto del proceso, que a los aspectos metodológicos; b) priorizar los enfoques multidisciplinarios y transdisciplinarios; c) vincular a los centros de capacitación y a las instituciones de educación superior con centros laborales, de investigación y otras organizaciones que se benefician del proceso de formación; d) incorporar con rapidez los adelantos científico-técnicos; e) utilizar cada vez en mayor medida las nuevas tecnologías educativas; y f) jerarquizar las formas organizativas que demandan un papel más activo del estudiante en el proceso de aprendizaje.

Igualmente, se recomienda que para favorecer un proceso de enseñanza-aprendizaje fluido, profundo, amplio, participativo, efectivo y eficiente, se incorporen en la metodología de enseñanza instrumentos de formación como: Clases magistrales, conferencias, video-conferencias, mesas redondas, estudios de casos, ejercicios de simulación, foros, ensayos y controles de lectura, trabajos de investigación o monografías finales, exposiciones y debates.

Por último, reseñamos como antecedente el trabajo realizado por el Ministerio de Planificación y Desarrollo (MPD, 2007) a través del Sistema Nacional de Adiestramiento (SNA), el cual desarrolla actualmente programas de capacitación dirigidos a la formación continua de los funcionarios públicos en áreas como: acción social, subsistemas y procesos de recursos humanos, procesos administrativos y fiscales, desarrollo supervisorio y gerencial, crecimiento y desarrollo personal, adiestramiento tecnológico, desarroIlo secretarial, apoyo y servicios, aspectos jurídicos y programas especiales.

Como se nota son programas de formación dirigidos al funcionario público en el ámbito técnico-administrativo, sin embargo, la necesidad de que la formación de los funcionarios públicos esté adaptada a las nuevas exigencias y realidades del país, ha demandado otras áreas y estrategias, por lo que el SNA, está llevando a cabo un proceso de formación por medio de talleres para los funcionarios públicos y las comunidades de integración.

Esta formación se está realizando a nivel nacional e incluye módulos sobre: 1) El Estado y la Sociedad Venezolana, 2) la Función Pública y 3) La Participación Ciudadana. Destaca en el planteamiento la combinación de temas éticos y políticos, tratados en el contexto específico de la función pública.

De estos módulos se desprenden talleres para fortalecer las relaciones Estado - Sociedad en las áreas de: Desarrollo Endógeno, Identidad Nacional, Promotores del Cambio Social e Introducción al Trabajo Comunitario.

\section{El perfil del funcionario para la participación popular}

Una aproximación del perfil del funcionario público requerido para la nueva gestión basada en la participación popu- 
Formación para la administración municipal basada en la participación popular protagónica Porras Medina, Ada Crisey y González Silva, Humberto J.

lar, puede tomar como referencia el perfil deseable para el gerente social planteado por Kliksberg (1992):

a) Capacidad para gerenciar complejidad

"A las nociones de probabilidad y riesgo se han sumado otras, como "desconocimiento" (Kliksberg, 1992:99), que destacan que hay variables incidentes que ni siquiera sabemos que existen. "Frente a la complejidad hay dos posibilidades. Una tradicional es la de "reprimir" la incertidumbre no admitiendo mayormente su existencia y repitiendo decisiones en función del pasado. Otra es tratar de enfrentarla preparando al gerente para manejarse en esa situación" (Kliksberg, 1992:99).

b) Orientación a la articulación social "Existen, entre otros riesgos, los del elitismo y la manipulación tecnocrática. Se puede tratar de articular desde arriba, del "laboratorio", o hacerlo más sofisticadamente con apariencias populistas pero intención manipulatoria. Ambas vías conducirán a resultados carentes de solidez y a legítimas "formaciones reactivas". El profesional debe estar preparado para, en cambio, actuar como favorecedor de procesos que deben surgir de sus mismos protagonistas, y ser conducidos por ellos" (Kliksberg, 1992:100).

c) Capacidades para la concertación La capacidad de operar proyectos multiinstitucionales, como muchos de los que nos toca adelantar como nación, tropieza con hechos preexistentes como el enfeudamiento, característico de los aparatos públicos, basado en búsquedas de poder de sectores burocráticos definidos, múltiples alinea- mientos de intereses, etc. El profesional deberá disponer de calidades especiales para "concertar" en estas condiciones.

d) Gerencia de frontera tecnológica Flexibilidad, trabajo por proyectos, rotación horizontal de recursos humanos, metas cambiantes, adaptabilidad, innovación, pensamiento estratégico, creación de condiciones para la innovación, acción en equipo de carácter interdisciplinario, participación, experimentación, riesgo... Son características exigidas a la nueva gestión, y casi opuestas a los usos dominantes en nuestra realidad y fomentados desde nuestra educación.

e)Formación para el compromiso

Requerimos funcionarios comprometidos con los propósitos de la construcción del poder popular y la construcción del socialismo bolivariano. Ello implica: "En lugar de una neutralidad sistemática (...) una identificación activa, entre otros aspectos, con la democratización, el cambio, el desarrollo humano integral sustentable" (Kliksberg, 1992:102).

Este compromiso ha de traducirse en el ejercicio activo de nuevos valores y la identificación con los cambios dirigidos a la transformación del pensamiento, que a su vez van acompañados de una dimensión muchas veces olvidada: la moral, tal como lo ha planteado el Presidente Chávez en sus discursos sobre el Tercer Motor Constituyente: Moral y Luces.

\section{Conclusiones}

La investigación documental permite caracterizar un conjunto de características indispensables a considerar en el 
diseño e implementación de una propuesta de formación dirigida a los funcionarios municipales, para impulsar una gestión municipal en función de la participación popular. Estas son:

1. Toda propuesta formativa debe partir de claras definiciones sobre lo que se aspira. Serán radicalmente distintas las propuestas que se funden sobre una visión funcional de la participación de aquellas que aspiran a la realización plena de la soberanía popular como eje de la construcción de una nueva sociedad. La discusión sobre los alcances de la participación involucra un debate sobre el poder, sus formas de ejercicio y sus manifestaciones en la cotidianidad. El cambio de poder y la construcción de la nueva sociedad implican compromiso y requieren de la revisión profunda de los supuestos acerca del cambio.

2. Lo anterior se traduce en que la formación tiene necesariamente una dimensión ética y política, las cuales plantean retos mayores a las propuestas de formación, pues éstas no pueden reducirse a los aspectos jurídicos e instrumentales.

3. El conocimiento profundo de la administración pública, sus claras diferencias con la gerencia privada, así como del nuevo papel del Estado como promotor del Poder Popular que aspira estar al servicio de éste resultan centros ineludibles para toda propuesta de formación que pretenda efectivamente contribuir a un cambio hacia gestiones municipales fundadas en la participación.

4. Es indispensable que la formación para la participación involucre tanto a las comunidades como a los funcionarios, ya que la acción de éstos últimos sin el desarrollo de las competencias requeridas puede incluso resultar contraproducente para la participación popular.

5. La formación debe fundamentarse en un diagnóstico cuidadoso de las necesidades apreciando las experiencias existentes, sean éstas exitosas o no.

6. Es necesario trabajar para la fundamentación de las propuestas en la sistematización de experiencias de participación que den cuenta de los aciertos y desaciertos en su promoción.

7. Es imprescindible que las propuestas formativas busquen articulación entre sí para poder compartir experiencias, materiales y recursos. La debilidad del Estado en su capacidad para la formación y la participación solo puede ser superada mediante la conjugación de esfuerzos.

8. Las propuestas formativas deben enfocar sus esfuerzos en el aprendizaje mutuo y en las características de los participantes, para lo cual es necesario incorporar una combinación de estrategias metodológicas, particularmente aquellas que faciliten el diálogo entre funcionarios y comunidades, la reflexión compartida y la experimentación de cursos de acción.

\section{Referencias Bibliográficas}

Álvarez F., Oneida; Arellano P., Félix G.; Ramos Martino, Eira (2005). Proyecto de actualización curricular en materia de integración para los cursos de licenciaturas, postgrados y capacitación de funcionarios públicos en la comunidad andina. Infor- 
Formación para la administración municipal basada en la participación popular protagónica Porras Medina, Ada Crisey y González Silva, Humberto J.

me Final. CLAD, CAF. Disponible en: http://www.clad.

org.ve/fulltext/0051700.pdf [Consulta 20-03-2007]

Aristizábal, Henao y otros (2001). Sistematización de experiencias de veedurías ciudadanas y control social en la Administración Pública. Estrategias de Participación Ciudadana. Trabajo de Grado para optar al título de Trabajador Social. Universidad de la Salle. Facultad de Trabajo Social. Bogotá D.C.

Cabrero Mendoza, Enrique (1995). La nueva gestión municipal en México. Análisis de experiencias innovadoras en gobiernos locales. Centro de Investigación y Docencia Económicas Miguel Angel Porrúa.

Cortázar, Juan Carlos y Lecaros, Carla (2003). La viabilidad de las prácticas de participación ciudadana en la gestión municipal. El caso del presupuesto participativo de Villa EI Salvador (Perú, 2000-2002). Instituto Interamericano para el Desarrollo Social (INDES), Washington D.C. Disponible en: http://indes.iadb.org/eve/ 1495/VillaSalvador.pdf [Consulta 2303-2007].

Chávez Frías, Hugo (2004a, Noviembre). TaIler de Alto Nivel "El Nuevo Mapa Estratégico". Intervenciones del Presidente de la República. Caracas.

Chávez Frías, Hugo (2004b, Septiembre). ¿Queremos acabar con la pobreza demos poder a los pobres? En apoyo a la propuesta Lula: Hambre Cero. Naciones Unidas.

Constitución de la República Bolivariana de Venezuela (1999). Gaceta Oficial $\mathbf{N}^{\circ}$ 5.453. Extraordinaria de fecha 24-022000.

Coraggio, José L. (2004). De la emergencia a la estrategia. Más allá del "alivio a la pobreza. Espacio Editorial, Buenos Aires. Espacio.
Corredor, Janna (2006, Mayo 02). Chávez destacó que consejos comunales son la vía para llegar al socialismo. Disponible: www.abn.info.ve

Delgado, José Gregorio y Quintin, Ramón (2006). Marco Jurídico e Institucional de la Participación Ciudadana en Venezuela. Módulo 1.

Díaz P., Nelson, Rivera M., Claudio y Kraushaar, Lilith (2002). "Nuevas Estrategias de Formación Profesional para la Modernización del Estado en Chile". Revista Venezolana de Gerencia. Abril - junio/año vol. 7, número 18. Universidad del Zulia. Maracaibo. Venezuela.

El Troudi, Haiman, Harnecker, Marta y BoniIla-molina, Luis (2005). Herramientas para la Participación. Venezuela: edición financiada por CVG.

Fisher, Tania (coord.) (1993). Poder local: governo e cidadania. Fundacao Getulio Vargas. Rio de Janeiro Brasil.

Fontdevila, Pablo A. (2002). Las nuevas tecnologías al servicio de la formación de administradores públicos. VII Congreso Internacional del CLAD sobre la Reforma del Estado y de la Administración Pública, Lisboa, Portugal. Disponible: http://unpan1.un.org. Consulta: [2007, Mayo]

Gallicchio, Enrique (2002). Conferencia electrónica - Empoderamiento y Desarrollo Local. Disponible: http://www. desarrollolocal.org. [Consulta: febrero 2007].

Guerrero, Omar (2005). Principios de la Administración Pública. Séptima lección. Las administraciones no estatales. Disponible: http://omega.ilce. edu.mx:3000/sites/csa/principio/leccion7.html. [Consulta: 2006, Mayo 27]

Iglesias y Tabares (2004). Formación en Administración Pública en América Latina, Canadá y Cuba: Particularidades y Tendencias [Documento en 
línea]. Disponible: http: www.uh.cu. [Consulta: 2006, mayo 27].

Kliksberg, Bernardo (Comp.) (1992). ¿Cómo enfrentar la pobreza? Aportes para la acción. Segunda edición revisada y ampliada. Grupo Editor Latinoamericano. Colección Estudios Políticos. Buenos Aire.

Llona, Mariana (2001). Gestión local participativa: del barrio a la ciudad. Disponible: http://www.unhabitat.org. [Consulta: 2006 Abril].

Melucci, Alberto (1999). "Teoría de la acción colectiva" En: Acción colectiva, vida cotidiana y democracia. México DF. Disponible: http://www.participacion. org.pe/biblio.htm. [Consulta: 2006 Mayo].

Millaleo, Salvador y Valdes, Marcos (2002). EI concepto de participación en el Programa Orígenes (Desarrollo Integral de Comunidades Indígenas).

República Bolivariana de Venezuela. Ley del Estatuto de la Función Pública. Gaceta Oficial № $\mathbf{3 7 . 5 5 2}$ del 06 de septiembre de 2002.

República Bolivariana de Venezuela. Ley Orgánica de la Administración Pública. Gaceta Oficial No 37.305 del 17 de Octubre de 2001.
República Bolivariana de Venezuela. Ley Orgánica del Poder Público Municipal. Gaceta Oficial $N^{\circ} \mathbf{3 8 . 2 0 4}$ del 8 de junio de 2005.

Rodríguez, Jorge (2007). Explosión del Quinto Motor, discurso pronunciado en Acto con motivo de la explosión del Quinto Motor Constituyente, en el Parque Los Caobos, Caracas, el 28 de febrero de 2007. Disponible en: http://www.vicepresidencia.gov.ve/ alocucion. asp?numn=11

SD Gateway. [Página Web en línea]. Disponible: http://www.sdgateway.net. [Consulta: 2006, Abril 15]

Tohá, Carolina y Solari, Ricardo (1996). La Modernización del Estado y la Gerencia Pública. Disponible: http:// www.fes.org.ar/. [Consulta: 2006, Mayo]

Stiglitz, J.E. (1989). The economic role of the state. Basil Backwell, Oxford.

Vargas, Carlos (1998). Desarrollo local y participación política en llo. Nuevas formas de articulación y representación social y política. Disponible: http://www.participacion.org.pe/biblio.htm. [Consulta: 2006 Mayo]. 